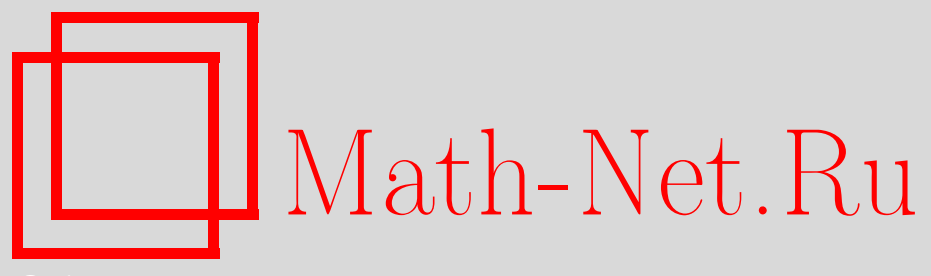

А. Ю. Веснин, Д. Реповш, Двусторонние оценки объемов прямоугольных гиперболических многогранников, Матем. заметки, 2011, том 89, выпуск 1, 12-18

DOI: https://doi.org/10.4213/mzm8922

Использование Общероссийского математического портала Math-Net.Ru подразумевает, что вы прочитали и согласны с пользовательским соглашением http: //www. mathnet.ru/rus/agreement

Параметры загрузки:

IP : 18.208 .226 .222

26 апреля 2023 г., 13:03:29

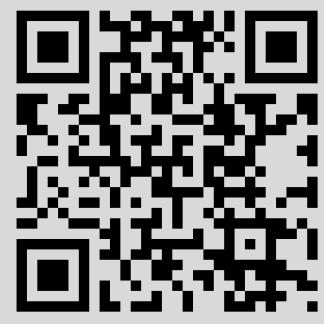


Том 89 выпуск 1 январь 2011

УДК 514

\title{
Двусторонние оценки объемов прямоугольных гиперболических многогранников
}

\author{
А. Ю. Веснин, Д. Реповш
}

Для компактного прямоугольного многогранника $R$ в пространстве Лобачевского $\mathbb{H}^{3}$ обозначим через $\operatorname{vol}(R)$ его объем, а через $\operatorname{vert}(R)$ - число вершин. Верхние и нижние оценки величины $\operatorname{vol}(R)$ в терминах $\operatorname{vert}(R)$ были недавно получены Аткинсоном. Строя 2-параметрическое семейство многогранников, мы показываем, что асимптотическая верхняя оценка $5 v_{3} / 8$, где $v_{3}$ - объем правильного идеального тетраэдра в $\mathbb{H}^{3}$, является двойной предельной точкой для отношений $\operatorname{vol}(R) / \operatorname{vert}(R)$. Более того, мы улучшаем нижнюю оценку для случая $\operatorname{vert}(R) \leqslant 56$.

Библиография: 12 названий.

1. Прямоугольные многогранники в трехмерном пространстве Лобачевского. В каждом из возможных геометрических пространств прямоугольные многогранники являются весьма удобными "строительными блоками" для различных геометрических конструкций. Мы будем рассматривать многогранники в трехмерном пространстве Лобачевского $\mathbb{H}^{3}$. Необходимые и достаточные условия, при которых многогранник заданного комбинаторного типа реализуется как компактный выпуклый прямоугольный многогранник в $\mathbb{H}^{3}$, были описаны Погореловым в 1967 г. в самом первом номере "Математических заметок" [1]. Простейшим из компактных прямоугольных гиперболических многогранников является додекаэдр со всеми двугранными углами $\pi / 2$ (а следовательно, и всеми плоскими углами $\pi / 2$ ). Как и было бы естественно ожидать, такие многогранники играют весьма полезную роль при построении гиперболических 3-многообразий. Так, можно попытаться построить гиперболическое 3-многообразие, используя прямоугольный многогранник как его фундаментальный многогранник. Или же можно построить такое гиперболическое 3-многообразие, что его фундаментальная группа является подгруппой без кручения в группе Коксетера, порожденной отражениями в гранях прямоугольного многогранника [2]. Ниже мы рассматриваем только компактные многогранники, т.е. многогранники, не имеющие идеальных вершин.

Начнем с формулировок двух недавних результатов. Иноуе в [3] ввел две операции на прямоугольных многогранниках, названные им декомпозищия и реберная

Работа первого автора выполнена при поддержке Российского фонда фундаментальных исследований (грант № 09-01-00255) и интеграционного гранта СО РАН - УрО РАН. Работа второго автора выполнена при поддержке грантов P1-0292-0101, J1-9643-0101 и J1-2057-0101 Исследовательского агенства Словении.

(C) А. Ю. Веснин, Д. Реповш, 2011 
хирургия, и доказал, что многогранники Лёбелля (которые мы подробно обсудим ниже) являются универсальными в следующем смысле.

Теорема 1 [3; теорема 9.1]. Пусть $P_{0}$ - компактный прямоуголъный гиперболический многогранник. Тогда существует последовательность объединений попарно непересекающихся прямоугольных гиперболических многогранников $P_{1}, \ldots, P_{k}$ такая, что для $i=1, \ldots, k P_{i}$ получено из $P_{i-1}$ операцией декомпозиции или операцией реберной хирургии, а $P_{k}-$ множество многогранников Лёбелля. При этом

$$
\operatorname{vol}\left(P_{0}\right) \geqslant \operatorname{vol}\left(P_{1}\right) \geqslant \operatorname{vol}\left(P_{2}\right) \geqslant \cdots \geqslant \operatorname{vol}\left(P_{k}\right) .
$$

Аткинсон [4] оценил объем прямоугольного многогранника в терминах числа его вершин следующим образом.

Теорема 2 [4; теорема 2.3]. Если $P$ - компактный прямоуголъный гиперболический многогранник с $V$ вершинами, то

$$
(V-2) \cdot \frac{v_{8}}{32} \leqslant \operatorname{vol}(P)<(V-10) \cdot \frac{5 v_{3}}{8},
$$

где $v_{8}$ - обгем правильного идеального октаэдра, а $v_{3}$ - обгем правильного идеального тетраэдра. Существует последовательность компактных многогранников $P_{i}$, где $P_{i}$ имеет $V_{i}$ вершин таких, что $\operatorname{vol}\left(P_{i}\right) / V_{i}$ стремится $\kappa 5 v_{3} / 8$ при $i$ стремящемся $\kappa$ бесконечности.

Семейство многогранников $P_{i}$, предложенное Аткинсоном, описано в доказательстве [4; предложение 6.4].

В этой заметке мы покажем, что многогранники Лёбелля могут служить в качестве подходящего семейства, на котором реализуется верхняя оценка. Тем самым, многогранники Лёбелля играют важную роль не только в теореме 1, но и в теореме 2 .

Обозначим через $\operatorname{vert}(R)$ число вершин прямоугольного многогранника $R$. Следующий результат показывает, что величина $5 v_{3} / 8$ является двойной предельной точкой в том смысле, что она является предельной точкой для предельных точек последовательностей отношений $\operatorname{vol}(R) / \operatorname{vert}(R)$.

ТЕОРема 3. Для любого иелого $k \geqslant 1$ существует последовательность компактных прямоуголъных гиперболических многогранников $R_{k}(n)$ таких, что

$$
\lim _{n \rightarrow \infty} \frac{\operatorname{vol}\left(R_{k}(n)\right)}{\operatorname{vert}\left(R_{k}(n)\right)}=\frac{k}{k+1} \cdot \frac{5 v_{3}}{8} .
$$

Как будет видно из доказательства, многогранники $R_{1}(n)$ являются многогранниками Лёбелля, а многогранники $R_{k}(n), k>1,-$ башнями, составленными из них.

Кроме того, в следствии 4 мы улучшим нижние оценки из теоремы 2 в случае, когда $\operatorname{vert}(R) \leqslant 56$.

2. Многогранники и многообразия Лёбелля. Многогранники Лёбелля были введены в [2] как обобщение прямоугольного 14-гранника, использовавшегося в работе [5].

Напомним, что давая положительный ответ на вопрос о существовании пространственных форм Клиффорда-Клейна (т.е. замкнутых многообразий) постоянной отрицательной кривизны, Лёбелль [5] построил в 1931 г. первый пример замкнутого 
ориентируемого гиперболического 3-многообразия. Это многообразие было получено склеиванием восьми экземпляров прямоугольного 14-гранника (обозначенного через $R(6)$ и изображенного на рис. 1). Его верхнее и нижнее основания являются правильными шестиугольниками, а боковая поверхность образована 12 пятиугольниками, расположенными аналогично боковой поверхности додекаэдра. Очевидно, $R(6)$ можно воспринимать как обобщение прямоугольного додекаэдра при замене пятиугольных верхнего и нижнего оснований шестиугольными.

Как показано в [2], додекаэдр и многогранник $R(6)$ являются элементами следующего семейства многогранников. Для каждого $n \geqslant 5$ рассмотрим прямоугольный многогранник $R(n)$ в $\mathbb{H}^{3}$, имеющий $(2 n+2)$ граней, две из которых (рассматриваемые как его верхнее и нижнее основания) являются правильными $n$-угольниками. Его боковая поверхность образована $2 n$ пятиугольниками, расположенными по аналогии с пятиугольниками на боковой поверхности додекаэдра. В частности, $R(5)$ - это прямоугольный додекаэдр (см. рис. 1). Существование многогранников $R(n)$ в $\mathbb{H}^{3}$ легко устанавливается благодаря теореме Погорелова [1] или теореме Андреева [6].
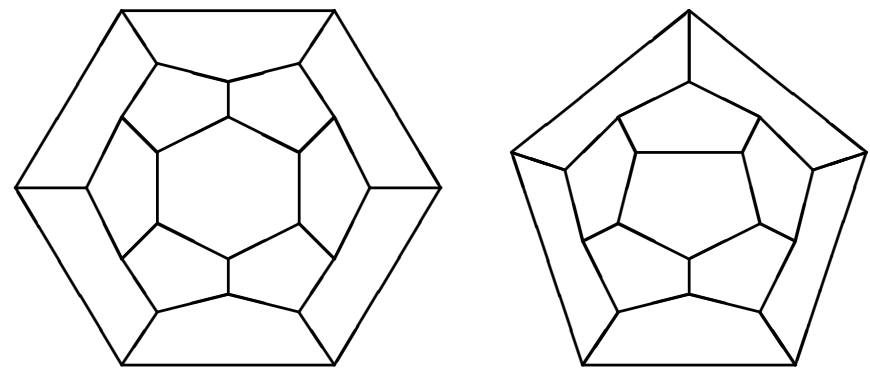

Рис. 1. Многогранники $R(6)$ и $R(5)$

Подход, предложенный в [2], позволяет строить как ориентируемые, так и неориентируемые замкнутые гиперболические 3-многообразия из восьми экземпляров любого компактного прямоугольного гиперболического многогранника. А именно, каждая раскраска граней прямоугольного многогранника в четыре цвета такая, что никакие две грани одного цвета не имеют общее ребро, задает состоящую из сохраняющих ориентацию изометрий подгруппу без кручения индекса восемь в группе Коксетера исходного многогранника.

Таким образом, каждая четырехцветная раскраска задает ориентируемое гиперболическое 3-многообразие, получаемое из восьми экземпляров прямоугольного многогранника. Этот подход также позволяет строить неориентируемые гиперболические 3-многообразия, но при этом требуется от пяти до семи цветов.

В [2] отмечено, что многообразие, построенное Лёбеллем в 1931 г., может быть задано четырехцветной раскраской многогранника $R(6)$. Кроме того, для каждого $n \geqslant 5$ явно указаны примеры как ориентируемых, так и неориентируемых многообразий, строящихся из восьми экземпляров $R(n)$. Замкнутые ориентируемые гиперболические 3 -многообразия, кодируемые четырехцветными раскрасками многогранников $R(n), n \geqslant 5$, получили название многообразия Лёбелля. (Отметим, что для заданного $n$ число таких многообразий может быть отлично от единицы.) Многогранники $R(n)$ естественно называть многогранниками Лёбелля. 
Свойства многообразий Лёбелля изучались довольно интенсивно: точные формулы объемов получены в [7] и [8]; инвариантные поля следов фундаментальных групп многообразий и их арифметичность были численно исследованы в [9]; в [10] многие многообразия описаны как двулистные разветвленные накрытия 3-сферы; двусторонние оценки сложности многообразий Лёбелля получены в [11], [12].

Для выражения объемов гиперболических многогранников традиционно используется восходящая к работе Лобачевского 1832 г. следующая функиия Лобачевского:

$$
\Lambda(x)=-\int_{0}^{x} \log |2 \sin (t)| d t .
$$

Поскольку каждое многообразие Лёбелля, соответствующее параметру $n$, получается склеиванием изометриями восьми экземпляров многогранников $R(n)$, формула объема многообразий Лёбелля, установленная в [7], влечет следующую формулу для $\operatorname{vol}(R(n))$.

Теорема 4. Для всех $n \geqslant 5$ имеет место формула

$$
\operatorname{vol}(R(n))=\frac{n}{2}\left(2 \Lambda\left(\theta_{n}\right)+\Lambda\left(\theta_{n}+\frac{\pi}{n}\right)+\Lambda\left(\theta_{n}-\frac{\pi}{n}\right)+\Lambda\left(\frac{\pi}{2}-2 \theta_{n}\right)\right),
$$

где

$$
\theta_{n}=\frac{\pi}{2}-\arccos \left(\frac{1}{2 \cos (\pi / n)}\right)
$$

Легко проверить, что $\theta_{n} \rightarrow \pi / 6$ и

$$
\frac{\operatorname{vol}(R(n))}{n} \rightarrow \frac{5 v_{3}}{4}
$$

при $n \rightarrow \infty$. Здесь мы воспользовались тем, что $v_{3}=3 \Lambda(\pi / 3)=2 \Lambda(\pi / 6)$. Более того, асимптотическое поведение объемов многообразий Лёбелля было установлено в [12; предложение 2.10]. Отсюда легко следует описание асимптотического поведения $\operatorname{vol}(R(n))$ при $n$ стремящемся к бесконечности.

ПреДЛОЖЕНИЕ 1. Следующие неравенства имеют место для достаточно больuux $n$ :

$$
\frac{5 v_{3}}{4} \cdot n-\frac{17 v_{3}}{2 n}<\operatorname{vol}(R(n))<\frac{5 v_{3}}{4} \cdot n .
$$

Поскольку $\operatorname{vert}(R(n))=4 n$, мы получаем

СлЕДСТВиЕ 1. Следующие неравенства имеют место для достаточно больuux $n$ :

$$
\frac{5 v_{3}}{16}-\frac{17 v_{3}}{8 n^{2}}<\frac{\operatorname{vol}(R(n))}{\operatorname{vert}(R(n))}<\frac{5 v_{3}}{16} .
$$

3. Доказательство теоремы 3. Мы будем использовать многогранники Лёбелля $R(n)$ как блоки для построения прямоугольных многогранников с нужными свойствами. Представим многогранник $R(n)$ его боковой поверхностью, как это сделано на рис. 2 для многогранников $R(6)$ и $R(5)$, подразумевая, что левый и правый края должны быть отождествлены. 

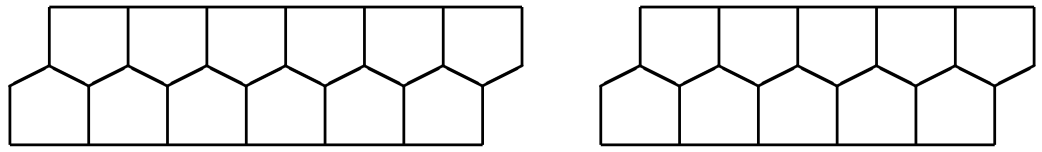

Рис. 2. Многогранники $R(6)$ и $R(5)$

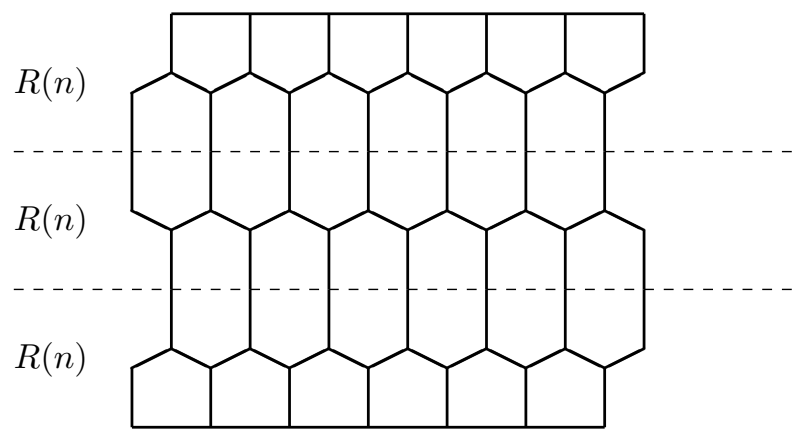

Рис. 3. Многогранник $R_{3}(6)$

Для целого $k \geqslant 1$ обозначим через $R_{k}(n)$ многогранник построенный из $k$ экземпляров многогранника $R(n)$ склеиванием их по $n$-угольным граням подобно башне. В частности, $R_{1}(n)=R(n)$. Многогранник $R_{3}(6)$ представлен на рис. 3 .

Очевидно, $R_{k}(n)$ является прямоугольным многогранником с $n$-угольными верхним и нижним основаниями и боковой поверхностью, образованной $2 n$ пятиугольниками и $(k-1) \cdot n$ шестиугольниками.

Поскольку $\operatorname{vol}\left(R_{k}(n)\right)=k \cdot \operatorname{vol}(R(n))$, предложение 1 влечет, что для достаточно больших $n$ выполнено

$$
k \cdot \frac{5 v_{3}}{4} \cdot n-k \cdot \frac{17 v_{3}}{2 n}<\operatorname{vol}\left(R_{k}(n)\right)<k \cdot \frac{5 v_{3}}{4} \cdot n .
$$

Поскольку vert $R_{k}(n)=(2 k+2) n$, получаем

$$
\frac{k}{k+1} \cdot \frac{5 v_{3}}{8}-\frac{k}{k+1} \cdot \frac{17 v_{3}}{4 n^{2}}<\frac{\operatorname{vol}\left(R_{k}(n)\right)}{\operatorname{vert}\left(R_{k}(n)\right)}<\frac{k}{k+1} \cdot \frac{5 v_{3}}{8} .
$$

Таким образом, семейство прямоугольных многогранников $R_{k}(n)$ таково, что для каждого целого $k \geqslant 1$ выполнено

$$
\lim _{n \rightarrow \infty} \frac{\operatorname{vol}\left(R_{k}(n)\right)}{\operatorname{vert}\left(R_{k}(n)\right)}=\frac{k}{k+1} \cdot \frac{5 v_{3}}{8},
$$

и верхняя оценка $5 v_{3} / 8$ является двойной предельной точкой в том смысле, что она является предельной точкой для указанных выше предельных точек при $k \rightarrow \infty$ :

$$
\lim _{k, n \rightarrow \infty} \frac{\operatorname{vol}\left(R_{k}(n)\right)}{\operatorname{vert}\left(R_{k}(n)\right)}=\frac{5 v_{3}}{8} .
$$

Таким образом, теорема доказана. 
4. Другие оценки объемов. Поскольку 1-скелет прямоугольного компактного гиперболического многогранника $P$ является трехвалентным плоским графом, из формулы Эйлера для многогранника следует, что

$$
V=2 F-4,
$$

где $V$ - число вершин многогранника $P$, а $F$ - число его граней. Более того, из формулы Эйлера следует, что $P$ имеет, по крайней мере, 12 граней (это число граней соответствует додекаэдру). Таким образом, теорема 2 влечет следующий результат.

СлЕДСТВИЕ 2. Пусть $P$ - компактный прямоуголъный гиперболический многогранник с F гранями. Тогда

$$
(F-3) \cdot \frac{v_{8}}{16} \leqslant \operatorname{vol}(P)<(F-7) \cdot \frac{5 v_{3}}{4} .
$$

Напомним, что константы $v_{3}$ и $v_{8}$ имеют следующий вид:

$$
\begin{aligned}
& v_{3}=3 \Lambda\left(\frac{\pi}{3}\right)=1.0149416064096535 \ldots, \\
& v_{8}=8 \Lambda\left(\frac{\pi}{4}\right)=3.663862376708876 \ldots
\end{aligned}
$$

Поскольку площадь прямоугольного $n$-угольника равна $\pi / 2 \cdot(n-4)$, площадь боковой поверхности компактного прямоугольного гиперболического многогранника $P$ с $F$ гранями равна $\pi \cdot(F-6)$. Таким образом, следствие 2 влечет следующий результат.

СлЕДСТВИЕ 3. Пусть $P$ - компактный прямоуголъный гиперболический многогранник, площадъ боковой поверхности которого равна $S$. Тогда

$$
\left(\frac{S}{\pi}+3\right) \cdot \frac{v_{8}}{16} \leqslant \operatorname{vol}(P)<\left(\frac{S}{\pi}-1\right) \cdot \frac{5 v_{3}}{4} .
$$

Отметим, что теорема 4 может быть использована для доказательства того, что функция объема $\operatorname{vol}(R(n))$ является монотонно возрастающей функцией от $n$ (см. доказательства в [2] или [12]), и для вычисления объемов многогранников Лёбелля. В частности,

$$
\operatorname{vol}(R(5))=4.306 \ldots, \quad \operatorname{vol}(R(6))=6.203 \ldots, \quad \operatorname{vol}(R(7))=7.563 \ldots
$$

Вместе с теоремой 1 это влечет, что прямоугольным гиперболическим многогранником наименьшего объема является $R(5)$ (додекаэдр), а вторым по объему является многогранник $R(6)$. Так, если $P$ - компактный прямоугольный гиперболический многогранник, отличный от додекаэдра, то

$$
\operatorname{vol}(P) \geqslant 6.203 \ldots
$$

Таким образом, получаем

СлеДСТВИЕ 4. Пусть $P$ - компактный прямоуголъный гиперболический многогранник, отличный от додекаэдра, имеющий $V$ вершин и $F$ граней. Тогда

$$
\operatorname{vol}(P) \geqslant \max \left\{(V-2) \cdot \frac{v_{8}}{32}, 6.203 \ldots\right\}, \quad \operatorname{vol}(P) \geqslant \max \left\{(F-3) \cdot \frac{v_{8}}{16}, 6.203 \ldots\right\} .
$$

Оценки из следствия 4 улучшают нижние оценки из теоремы 2 для $V \leqslant 56$ и нижние оценки из следствия 2 для $F \leqslant 30$. 


\section{СПИСОК ЦИТИРОВАННОЙ ЛИТЕРАТУРЫ}

[1] А. В. Погорелов, "О правильном разбиении пространства Лобачевского", Матем. заметки, 1:1 (1967), 3-8.

[2] А. Ю. Веснин, "Трехмерные гиперболические многообразия типа Лебелля”, Сиб. матем. журн., 28:5 (1987), 50-53.

[3] T. Inoue, "Organizing volumes of right-angled hyperbolic polyhedra", Algebr. Geom. Topol., 8:3 (2008), 1523-1565.

[4] C. K. Atkinson, "Volume estimates for equiangular hyperbolic Coxeter polyhedra", Algebr. Geom. Topol., 9:2 (2009), 1225-1254.

[5] F. Löbell, "Beispiele geschlossener dreidimensionaler Clifford-Kleinscher Räume negativer Krümmung", Ber. Verh. Sächs. Akad. Leipzig, 83 (1931), 167-174.

[6] Е. М. Андреев, "О выпуклых многогранниках в пространствах Лобачевского", Maтем. сб., 81:3 (1970), 445-478.

[7] А. Ю. Веснин, "Объемы трехмерных гиперболических многообразий Лебелля", Maтем. заметки, 64:1 (1998), 17-23.

[8] A. D. Mednykh, A. Yu. Vesnin, "Löbell manifolds revised", Сиб. электрон. матем. изв., 4 (2007), 605-609.

[9] O. Antolín-Camarena, G. R. Maloney, R. K. W. Roeder, "Computing arithmetic invariants for hyperbolic reflection groups", Complex Dynamics. Families and Friends, A. K. Peters, Wellesley, MA, 2008, 597-631.

[10] А.Ю. Веснин, А. Д. Медных, “Трехмерные гиперэллиптические многообразия и гамильтоновы графы", Сиб. матем. журн., 40:4 (1999), 745-763.

[11] А. Ю. Веснин, С. В. Матвеев, К. Петронио, “Двусторонние оценки сложности многообразий Лёбелля”, Докл. РАН, 416:3 (2007), 295-297.

[12] S. Matveev, C. Petronio, A. Vesnin, "Two-sided asymptotic bounds for the complexity of some closed hyperbolic three-manifolds", J. Aust. Math. Soc., 86:2 (2009), 205-219.

\section{А. Ю. Веснин}

Поступило

Институт математики им. С. Л. Соболева СО РАН,

29.12.2009

г. Новосибирск

E-mail: vesnin@math.nsc.ru

\section{Д. Реповш}

University of Ljubljana, Словения

E-mail: dusan.repovs@guest.arnes.si 